\title{
Condition assessment of elastic bearing supports using vibration data
}

\author{
Moatasem M. Fayyadh, H. Abdul Razak
}

Health monitoring of engineering structures has gained a lot of

interest over the last few years. Many engineering structures suffer

damage and deterioration when exposed to various loading and

environmental changes during their lifetime. This seriously affects

structural performance and may even lead to catastrophic structural

failures. Thus, inspection and testing of structural components

for deterioration and damage is essential when deciding on

the maintenance and repair strategies for at-risk structures. A current

alternative approach to conventional structural testing methods

is dynamic testing, which acquires modal parameters and

relates these to the health status of a structure. The fundamental

idea underlying the dynamic approach is that modal parameters,

namely natural frequency, mode shape, and modal damping, are

functions of physical properties of the structure, such as mass,

damping, stiffness and the support conditions. Therefore, any

change in the physical properties or support conditions causes

detectable changes in the modal parameters. Elastic bearing pads

are widely used for supporting bridge girders, and as base isolation of tall buildings to reduce seismic demand. The bearings are exposed to various loading conditions and environmental changes

which cause deterioration of its stiffness with time. Monitoring

of changes in elastic bearing stiffness is very important in ensuring

timely maintenance or replacement to prevent occurrence of any

serious damage to the structure.

Several studies on the use of the modal parameters as an indicator

for damage identification have been conducted. Some of

these studies were concerned with issues related to use of these

modal parameters in determining the magnitude and localisation

of damage based on the relationship between dynamic and physical

properties, and concluded that modal parameters are good indicators

for damage detection [1-7]. Abdul Razak and Choi [8]

investigated the effect of steel corrosion on the natural frequencies

of $\mathrm{RC}$ beams. The authors concluded that the first natural frequency

for the RC beam with a lower corrosion level decreased

by $1 \%$, while for the $\mathrm{RC}$ beam with a higher corrosion level the decrease

was $4 \%$ and deduced that the bond at the steel-concrete

interface played a significant effect. Ismail [9] investigated the effect

of crack damage in $\mathrm{RC}$ beam on its natural frequencies. Flexural 
crack damage was induced by applying load at the mid-span

of the beam for three loading cycles. A comparison was made

based on the datum frequencies before applying the load to the

beams. It was found that the frequencies were reduced for all the applied loading cycles. Non-destructive evaluation procedure for identifying a crack, the location and size of the crack in one dimensional

beam type structure using the natural frequency data have

been proposed by Lee and Chung [10]. The application and

accuracy of the proposed procedure was demonstrated by comparing

with the results obtained by Rizos et al. [11] on crack detection

in a cantilever beam. Wang and Qiao [12] proposed a new damage

detection technique based on the irregularity profile of structural

mode shape. The results led to the conclusion that the proposed

technique required low measurement resolution and that the successful

detection of damage on the composite beam proved that

the proposed technique was capable of assessing both the location

and the size of the crack. Deraemaeker et al. [13] investigated

problems related to damage detection using output only vibration

measurement systems under changing environmental conditions.

The effect of changes in elastic bearing support conditions still requires further investigation. Many previous studies have used elastic bearing isolation systems to reduce seismic demand on structures, and many books have been written related to the design of these systems $[14,15]$. Various types of elastic bearings have been introduced as isolation systems. A number of studies have investigated the effectiveness of different types of elastic bearings, their material properties and their behaviour under different loading and environmental conditions with respect to the seismic responses of bridges [16-21].

Some studies have also been carried out on the effect of support conditions on the dynamic properties of structures. For example, Dai et al. [22] investigated the effect of stiffness of the rubber pads on dynamic characteristics of a base isolated bridge. The investigation was carried out on a model of a bridge that lacked isolation pads and for various values of shear modulus of the rubber. A direct relationship was found between rubber stiffness and natural frequencies, whereby increases in rubber stiffness resulted in increases in frequency. Carne et al. [23] investigated the effect of support stiffness and damping on measured modal frequencies and damping ratios using two different test models. The first model consisted of an extremely lightly damped beam that revealed changes in the measured modal frequency and damping. The second was a blade for a wind turbine, in which modal data were required to validate the analytical model of the blade. The changes in the measured modal parameters were significant and large enough that the support system 
was required to be taken into account when validating the analytical model of the blade. Investigation on the effect of stiffness of the supporting brace on the modal damping was done by Viola and Guidi [24]. The effect of support conditions on measured modal parameters was further investigated by Wolf [25] and Carne and Dohrmam [26], who validated the direct relationship between the support stiffness and the measured modal parameters obtained from previous studies.

The main objective of this study is to use the dynamic parameters i.e. natural frequency and mode shape, to detect the deterioration in the stiffness of elastic bearings supporting. Two cases are considered namely that of similar deterioration levels at both supports and differential deterioration at both supports, which has been never reported in literature. The sensitivity of each fundamental bending frequency and its mode shape are consider in order to ascertain the appropriate indicators for assessing the level of the deterioration in the elastic bearing support. This approach can be implemented for condition assessment of rubber bearing pads such as normally used for bridge decks. Moreover, from the results of this study it is envisage from the bending frequencies it is possible to differentiate and identify the loss of stiffness of the structural element i.e. cracking or of the girder, or due to deterioration of the elastic bearings.

Deterioration indicators

Two indicators are used in this study to monitor the elastic bearing deterioration level. The first is based on the change in the natural frequencies whilst the second is based on the change in the mode shape. Both natural frequency and mode shape are experimentally determined modal parameters which relate to the physical properties and the support stiffness of the structural elements (bridge girder). The natural frequency is an indicator for the global properties and it is usually used to indicate the damage severity. However the mode shape is an indicator for the local properties of the structural elements and it is usually used to indicate the damage severity at specific location along the structural element (bridge girder). The change in the mode shape indicates the change in the stiffness at a particular support for the structural element, while the change in the natural frequency indicates the overall change in the support stiffness.

Mode shape changes

The Modal Assurance Criteria (MAC) is used to calculate the change in the mode shape due to the support stiffness deterioration. MAC is a method used to ascertain configuration errors between 
experimental mode shapes and that predicted from the

finite element model [27]. This is a correlation between experimental

mode shapes and curve-fitted analytical model mode

shapes and the correlation for the ith element.

Full text available at :

http://www.sciencedirect.com/science/article/pii/S0950061811007203 